Communications in Physics, Vol. 26, No. 2 (2016), pp. 121-128

DOI: $10.15625 / 0868-3166 / 26 / 2 / 7446$

\title{
SPACETIMES WITH PSEUDOSYMMETRIC ENERGY-MOMENTUM TENSOR
}

\author{
SAHANOUS MALLICK ${ }^{\dagger}$ \\ Department of Mathematics, Chakdaha College, \\ P.O.- Chakdaha, Dist- Nadia, \\ West Bengal, India \\ UDAY CHAND DE \\ Department of Pure Mathematics, University of Calcutta, \\ 35, Ballygunge Circular Road, \\ Kolkata 700019 , \\ West Bengal, India \\ ${ }^{\dagger}$ E-mail: sahanousmallick@gmail.com
}

Received 25 November 2015

Accepted for publication 09 June 2016

\begin{abstract}
The object of the present paper is to introduce spacetimes with pseudosymmetric energymomentum tensor. In this paper at first we consider the relation $R(X, Y) \cdot T=f Q(g, T)$, that is, the energy-momentum tensor $T$ of type $(0,2)$ is pseudosymmetric. It is shown that in a general relativistic spacetime if the energy-momentum tensor is pseudosymmetric, then the spacetime is also Ricci pseudosymmetric and the converse is also true. Next we characterize the perfect fluid spacetime with pseudosymmetric energy-momentum tensor. Finally, we consider conformally flat spacetime with pseudosymmetric energy-momentum tensor.
\end{abstract}

Keywords: perfect fluid spacetime, Einstein's field equation, energy-momentum tensor, pseudosymmetric energy-momentum tensor.

Classification numbers: [The Mathematics Subject Classification(2010)] 53B30, 53C50, 53C80.

\section{INTRODUCTION}

General relativity flows from the Einstein's equation which implies that the energymomentum tensor is of vanishing divergence. This requirement of the energy-momentum tensor is satisfied if this tensor is covariant constant, that is, $\nabla T=0$, where $\nabla$ denotes the operator of covariant differentiation with respect to the metric tensor $g$. In the general theory of relativity, energy-momentum tensor plays an important role and the condition on energy-momentum (C)2016 Vietnam Academy of Science and Technology 
tensor for a perfect fluid spacetime changes the nature of spacetime [1]. In a recent paper [2] Chaki and Roy studied general relavistic spacetime with covariant constant energy-momentum tensor. Several authors studied spacetimes in several ways such as m-Projectively flat spacetimes by Zengin [3], generalized quasi-Einstein spacetimes by Güler and Demirbağ [4], generalized Robertson-Walker spacetimes by Arslan et al. [5] and many others. The spacetime of general relativity and cosmology is regarded as a connected 4-dimensional semi-Riemannian manifold $\left(M^{4}, g\right)$ with Lorentzian metric $g$ with signature $(-,+,+,+)$. The geometry of Lorentz manifold begins with the study of causal character of vectors of the manifold. It is due to this causality that Lorentz manifold becomes a convenient choice for the study of general relativity. Indeed by basing its study on Lorentzian manifold the general theory of relativity opens the way to the study of global questions about it ( [6-10]) and many others.

Let $\left(M^{n}, g\right)(n \geq 3)$ be a semi-Riemannian manifold with (not necessarily definite) metric $g$. We denote by $R$ and $S$ the Riemann-Christoffel curvature tensor and the Ricci tensor of $\left(M^{n}, g\right)$ respectively. For a tensor $P$ of type $(0, k)$ on $\left(M^{n}, g\right), k \geq 1$, we define the tensor fields $R \cdot P$ and $Q(g, P)$ by the formulas

$$
\begin{array}{r}
(R \cdot P)\left(X_{1}, X_{2} \ldots, X_{k} ; X, Y\right)=(R(X, Y) \cdot P)\left(X_{1}, X_{2}, \ldots, X_{k}\right) \\
=-P\left(R(X, Y) X_{1}, X_{2}, \ldots, X_{k}\right)-\ldots \\
-P\left(X_{1}, X_{2}, \ldots, X_{k-1}, R(X, Y) X_{k}\right),
\end{array}
$$

and

$$
\begin{array}{r}
Q(g, P)\left(X_{1}, X_{2}, \ldots, X_{k} ; X, Y\right)=\left(\left(X \wedge_{g} Y\right) \cdot P\right)\left(X_{1}, X_{2}, \ldots, X_{k}\right) \\
=-P\left(\left(X \wedge_{g} Y\right) X_{1}, X_{2}, \ldots, X_{k}\right)-\ldots \\
-P\left(X_{1}, X_{2}, \ldots, X_{k-1},\left(X \wedge_{g} Y\right) X_{k}\right),
\end{array}
$$

where $R(X, Y)$ and $X \wedge_{g} Y$ are derivations of the algebra of the tensor fields on $\left(M^{n}, g\right)$ respectively and $X_{1}, X_{2}, \ldots, X_{k}, X, Y \in \chi(M), \chi(M)$ being the Lie algebra of vector fields on $\left(M^{n}, g\right)$ and $Q(g, P)$ is the Tachibana tensor. These derivations are extensions of the endomorphisms $R(X, Y)$ and $X \wedge_{g} Y$ of $\chi(M)$ defined by

$$
R(X, Y) Z=\nabla_{X} \nabla_{Y} Z-\nabla_{Y} \nabla_{X} Z-\nabla_{[X, Y]} Z
$$

and

$$
(X \wedge Y) Z=g(Y, Z) X-g(Z, X) Y,
$$

respectively. The tensor field $Q(g, P)$ for $P=R$, was introduced in [11].

A semi-Riemannian manifold is said to be pseudosymmetric [12] if at every point of the manifold $M$ the tensors $R \cdot R$ and $Q(g, R)$ are linearly dependent, where $R$ is the Riemann-Christoffel curvature of type $(1,3)$ and $Q(g, R)$ is defined by

$$
\begin{aligned}
Q(g, R)(U, V, W ; X, Y)= & \left(X \wedge_{g} Y\right) R(U, V) W-R\left(\left(X \wedge_{g} Y\right) U, V\right) W \\
& \left.-R\left(U, X \wedge_{g} Y\right) V\right) W-R(U, V)\left(X \wedge_{g} Y\right) W .
\end{aligned}
$$

This is equivalent to

$$
R \cdot R=L_{R} Q(g, R),
$$

which holds on the set $U_{R}=\{x \in M: Q(g, R) \neq 0$ at $x\}$, where $L_{R}$ is some function on $U_{R}$. If $R \cdot R=0$, then the manifold $M$ is called semisymmetric. Every semisymmetric manifold is pseudosymmetric but the converse statement is not true. If $\nabla R=0$, then the manifold $M$ is called 
locally symmetric. It is trivial that if the manifold $M$ is locally symmetric then it is semisymmetric [13]. But a semisymmetric space is not necessarily locally symmetric. A fundamental study on such manifolds was made by Szabó $[14,15]$ and Kowalski [16]. In this connection we can mention the book of Boeckx, Kowalski and Vanhecke [17] and the references there in. Recently, pseudosymmetric spacetimes have been studied by several authors such as Defever et al. [18], Haesen et al. [19] and Deszcz et al. [20].

A semi-Riemannian manifold is said to be Ricci pseudosymmetric [12] if at every point of the manifold $M$ the tensors $R \cdot S$ and $Q(g, S)$ are linearly dependent, where $S$ denotes the Ricci tensor of type $(0,2)$. This is equivalent to

$$
R \cdot S=L_{S} Q(g, S),
$$

which holds on the set $U_{S}=\left\{x \in M: S \neq \frac{r}{n} g\right.$ at $\left.x\right\}$, where $L_{S}$ is some function on $U_{S}$. Every pseudosymmetric manifold is Ricci pseudosymmetric, but the converse statement is not true. If $R$. $S=0$, then the manifold $M$ is called Ricci semisymmetric [21]. Every semisymmetric manifold is Ricci semisymmetric, but the converse statement is not true. Every Ricci semisymmetric manifold is Ricci pseudosymmetric, but the converse statement is not true.

A semi-Riemannian manifold is said to be a manifold of quasi-constant curvature [22] if the curvature tensor $R$ of type $(1,3)$ satisfies the following condition:

$$
\begin{array}{r}
R(X, Y) Z=\lambda[g(Y, Z) X-g(X, Z) Y]+\mu[A(Y) A(Z) X-A(X) A(Z) Y \\
+g(Y, Z) A(X) \rho-g(X, Z) A(Y) \rho],
\end{array}
$$

where $\lambda, \mu$ are non-zero scalars and $A$ is a non-zero 1 -form defined by

$$
g(X, \rho)=A(X),
$$

for all $X$ and $\rho$ is a unit timelike vector field.

In 2015, De and Velimirović [23] generalized the result of Chaki and Roy [2] by considering the spacetime with semisymmetric energy-momentum tensor. Motivated by the above result, in the present paper we study spacetimes with pseudosymmetric energy-momentum tensor. Cosmology is the study of the whole universe as a physical system: what is its matter-energy content? How is this content organized? What is its history? How will it evolve in the future? But the study of cosmology depends crucially on our understanding of the gravitational interaction. Consequently, the proper framework for cosmology is General Relativity. The solution of Einstein's field equation describes the whole universe because it describes the whole spacetime. Also Einstein's field equation consists of energy-momentum tensor, Ricci tensor and metric tensor. Thus in this paper we have introduced pseudosymmetric energy-momentum tensor and study the spacetimes related with such a type of energy-momentum tensor. The pseudosymmetric energy-momentum tensor characterizes perfect fluid spacetime given by Theorem II.2 and conformally flat perfect fluid spacetime given by Theorem III.1. The results of Theorem II.2 and Theorem III.1 are important and interesting for physical aspects. In the litarature this concept is not available. This is the first time we introduce pseudosymmetric energy-momentum tensor in general relativistic spacetimes.

It is well known that warped product spacetimes form a very important subclass of the class of all spacetimes. It is known that [24] if $M_{1}=I$ is an open interval in $\mathbb{R}$, considered with its standard metric, let $F$ be a positive smooth function on $I$ and $M_{2}$ be a Riemannian manifold, then the manifold $M_{1} \times_{F} M_{2}$ is pseudosymmetric, provided $M_{2}$ is a space of constant curvature. This result 
implies that every Robertson-Walker spacetime is pseudosymmetric. The Schwarzschild spacetime and some of its generalizations (Reissner-Nordström and Kottler spacetimes) are pseudosymmetric and not semisymmetric. The Schwarzschild spacetimes represent the spherically symmetric, empty spacetimes outside nonrotating, spherically symmetric bodies. Since Sun and planets are assumed to be slowly rotating and approximately spherically symmetric, the Schwarzschild spacetimes may be used to model the gravitational fields outsite of these bodies. These spacetimes may also be used to model the gravitational fields outside of dead (i.e., nonrotating) black holes.

In this paper at first we consider the relation $R(X, Y) \cdot T=f Q(g, T)$, that is, the energymomentum tensor $T$ of type $(0,2)$ is pseudosymmetric, where the energy-momentum tensor $T$ represents the distribution of matter. It is shown that in a general relativistic spacetime if the energy-momentum tensor is pseudosymmetric, then the spacetime is also Ricci pseudosymmetric and the converse is also true. Next in Section II, we characterize the perfect fluid spacetime with pseudosymmetric energy-momentum tensor. Finally, we consider conformally flat spacetime with pseudosymmetric energy-momentum tensor.

\section{GENERAL RELATIVISTIC SPACETIMES WITH PSEUDOSYMMETRIC ENERGY-MOMENTUM TENSOR}

Let $\left(M^{4}, g\right)$ be a general relativistic spacetime and $T$ denote the $(0,2)$ type energy-momentum tensor. In this section we suppose that

$$
R(X, Y) \cdot T=f Q(g, T),
$$

where $R(X, Y)$ acts as a derivation on the tensor $T$ and $Q(g, T)$ is the Tachibana tensor.

We define the tensors $R \cdot T$ and $Q(g, T)$ as follows:

$$
(R \cdot T)(X, Y ; U, V)=-T(R(X, Y) U, V)-T(U, R(X, Y) V)
$$

and

$$
Q(g, T)(X, Y ; U, V)=-T\left(\left(X \wedge_{g} Y\right) U, V\right)-T\left(U,\left(X \wedge_{g} Y\right) V\right),
$$

where $\left(X \wedge_{g} Y\right) Z=g(Y, Z) X-g(X, Z) Y$.

Here,

$$
\begin{array}{r}
-T\left(\left(X \wedge_{g} Y\right) U, V\right)-T\left(U,\left(X \wedge_{g} Y\right) V\right)=-g(Y, U) T(X, V) \\
+g(X, U) T(Y, V)-g(Y, V) T(U, X) \\
+g(X, V) T(U, Y) .
\end{array}
$$

Then Einstein's equation can be written as

$$
S(X, Y)-\frac{r}{2} g(X, Y)=k T(X, Y),
$$

where $k$ is the gravitational constant.

Now

$$
(R(X, Y) \cdot T)(U, V)=f Q(g, T)
$$


implies that

$$
\begin{aligned}
-T(R(X, Y) U, V)-T(U, R(X, Y) V)=f[ & -g(Y, U) T(X, V) \\
& +g(X, U) T(Y, V)-g(Y, V) T(U, X) \\
& +g(X, V) T(U, Y)] .
\end{aligned}
$$

Using (12) in (13) yields

$$
\begin{aligned}
-S(R(X, Y) U, V)-S(U, R(X, Y) V)=f[ & -g(Y, U) S(X, V) \\
& +g(X, U) S(Y, V)-g(Y, V) S(U, X) \\
& +g(X, V) S(U, Y)] .
\end{aligned}
$$

That is,

$$
-S(R(X, Y) U, V)-S(U, R(X, Y) V)=f\left[-S\left(\left(X \wedge_{g} Y\right) U, V\right)-S\left(U,\left(X \wedge_{g} Y\right) V\right)\right],
$$

which means that

$$
R(X, Y) \cdot S=f Q(g, S)
$$

Thus we can state the following:

Theorem II.1. A general relativistic spacetime with pseudosymmetric energy-momentum tensor is Ricci pseudosymmetric and vice-versa.

If $f=0$, then we get $R \cdot T=0$, which implies that the energy-momentum tensor is semisymmetric. Therefore we have the following:

Corollary 1. A general relativistic spacetime with semisymmetric energy-momentum tensor is Ricci semisymmetric.

The above corollary has been proved by De and Velimirović [23].

Next we consider perfect fluid spacetime whose energy-momentum tensor is pseudosymmetric.

The energy-momentum tensor is said to describe a perfect fluid [25] if

$$
T(X, Y)=(\sigma+p) A(X) A(Y)+p g(X, Y),
$$

where $\sigma$ is the energy density and $p$ is the isotropic pressure of the fluid, $A$ is a non-zero 1-form such that

$$
g(X, \rho)=A(X),
$$

for all $X, \rho$ being the velocity vector field of the fluid which is a time-like vector, that is, $g(\rho, \rho)=$ $A(\rho)=-1$.

Then from (12) and (15) we get

$$
S(X, Y)=\alpha g(X, Y)+\beta A(X) A(Y),
$$

where $\alpha=\frac{r}{2}+k p$ and $\beta=k(\sigma+p)$.

Taking a frame field after contraction over $X, Y$, we get from (16) that

$$
r=k(\sigma-3 p),
$$

where $r=\sum \varepsilon_{i} S\left(e_{i}, e_{i}\right), \varepsilon_{i}=g\left(e_{i}, e_{i}\right)= \pm 1$. 
Suppose $R(X, Y) \cdot T=f Q(g, T)$. Then from Theorem II.2 we obtain $R(X, Y) \cdot S=f Q(g, S)$. Hence from (16) it follows that

$$
\begin{array}{r}
\beta A(R(X, Y) U) A(V)+\beta A(U) A(R(X, Y) V)=\beta g(Y, U) A(X) A(V) \\
-\beta g(X, U) A(Y) A(V)+\beta g(Y, V) A(U) A(X) \\
-\beta g(X, V) A(U) A(Y) .
\end{array}
$$

Putting $V=\rho$ and using $g(\rho, \rho)=A(\rho)=-1$ in (18) yields

$$
\beta A(R(X, Y) U)-\beta A(U) A(R(X, Y) \rho)=\beta g(Y, U) A(X)-\beta g(X, U) A(Y) .
$$

Since $A(R(X, Y) \rho)=g(R(X, Y) \rho, \rho)=0$, from the equation (19) it follows that

$$
\beta[A(R(X, Y) U)-g(Y, U) A(X)+g(X, U) A(Y)]=0,
$$

which implies that either

(i) $\beta=0$, or (ii) $A(R(X, Y) U)-g(Y, U) A(X)+g(X, U) A(Y)=0$.

Now (i) $\beta=0$ implies $\sigma+p=0$, since $k \neq 0$.

Also, $A(R(X, Y) U)-g(Y, U) A(X)+g(X, U) A(Y)=0$ implies $S(X, \rho)=3 A(X)$.

Substituting $Y=\rho$ in (16) and using $S(X, \rho)=3 A(X)$ yield $\alpha-\beta-3=0$, since the 1 -form $A$ is non-zero.

Now $\alpha-\beta-3=0$ gives $\kappa(\sigma+3 p)=-6$. Since $\kappa>0$, we must have $\sigma+3 p<0$.

Now $\sigma+p=0$ means the fluid behaves as a cosmological constant [26]. This is also termed as Phantom Barrier [27]. Now in a cosmology we know such a choice $\sigma=-p$ leads to rapid expansion of the spacetime which is now termed as inflation [28]. Also the inequality $\sigma+3 p<0$ shows the violation of cosmic strong energy condition(SEC). It means that the perfect fluid causing acceleration should violate SEC. As this energy(causing acceleration) is invisible, it is termed as dark energy [28].

Thus from the above discussion we can state the following theorem:

Theorem II.2. If the energy-momentum tensor of a perfect fluid spacetime is pseudosymmetric, then the spacetime is characterized by the following cases:

(i) The spacetime represents inflation and the fluid behaves as a cosmological constant.

(ii) The perfect fluid causing acceleration should violate strong energy condition.

This is also termed as dark energy.

\section{CONFORMALLY FLAT PERFECT FLUID SPACETIMES WITH PSEUDOSYMMETRIC ENERGY-MOMENTUM TENSOR}

In $\left(M^{4}, g\right)$ the conformal curvature tensor $C$ is defined by

$$
\begin{array}{r}
C(X, Y) Z=R(X, Y) Z-\frac{1}{2}[S(Y, Z) X-S(X, Z) Y \\
+g(Y, Z) Q X-g(X, Z) Q Y] \\
+\frac{r}{6}[g(Y, Z) X-g(X, Z) Y],
\end{array}
$$


where $Q$ is the Ricci operator defined by $g(Q X, Y)=S(X, Y), r$ is the scalar curvature. Let us suppose that the spacetime is conformally flat. Then from (20) we get

$$
\begin{array}{r}
R(X, Y) Z=\frac{1}{2}[S(Y, Z) X-S(X, Z) Y+g(Y, Z) Q X-g(X, Z) Q Y] \\
-\frac{r}{6}[g(Y, Z) X-g(X, Z) Y],
\end{array}
$$

From (16) we get

$$
Q X=\alpha X+\beta A(X) \rho .
$$

Now using (16) and (22) in (21) yields

$$
\begin{array}{r}
R(X, Y) Z=a[g(Y, Z) X-g(X, Z) Y]+b[A(Y) A(Z) X-A(X) A(Z) Y \\
+g(Y, Z) A(X) \rho-g(X, Z) A(Y) \rho],
\end{array}
$$

where $a=\alpha-\frac{r}{6}$ and $b=\frac{\beta}{2}$.

Equation (23) implies that the conformally flat perfect fluid spacetime is of quasi constant curvature [22].

By hypothesis $R \cdot T=f Q(g, T)$. Hence from Theorem II.1 we get $R \cdot S=f Q(g, S)$.

Also Yildiz, De and Cetinkaya [29] proved that in a Ricci pseudo symmetric quasi-Einstein manifold, the sum of the associated scalars is zero.

Taking a frame field after contraction over $Y, Z$ we get from (23) that

$$
S(X, W)=(3 a-b) g(X, W)+2 b A(X) A(W) .
$$

Here $3 a-b+2 b=0$ implies $p=-\frac{3}{2} \sigma$. This means that it represents the equation of state for barotropic fluid [30].

Thus we can state the following:

Theorem III.1. A conformally flat perfect fluid spacetime with pseudosymmetric energy-momentum tensor is a spacetime of quasi constant curvature and such a spacetime determines an equation of state for barotropic fluid.

\section{CONCLUSIONS}

The class of pseudosymmetric manifolds is a natural extension of the class of semisymmetric manifolds. Pseudosymmetric manifolds arose during the study of totally umbilical submanifolds in semisymmetric manifolds as well as from considerations of geodesic mappings onto semisymmetric manifolds. In the present paper we introduce spacetimes with pseudosymmetric energy-momentum tensor $T$, where $T$ represents the distribution of matter. It is shown that in a general relativistic spacetime if the energy-momentum tensor is pseudosymmetric, then the spacetime is also Ricci pseudosymmetric and the converse is also true. Next we characterize the perfect fluid spacetime with pseudosymmetric energy-momentum tensor. Finally, we consider conformally flat spacetime with pseudosymmetric energy-momentum tensor. Study of spacetimes with pseudosymmetric energy-momentum tensor is interesting and important for physical aspects.

\section{ACKNOWLEDGEMENT}

The authors wish to express their sincere thanks and gratitude to the referee for his/her valuable suggestions towards the improvement of the paper. 


\section{REFERENCES}

[1] H. Stephani and J. M. Stewart, General relativity: an introduction to the theory of the gravitational field cambridge university press, Camridge, 1982.

[2] M. C. Chaki and S. Ray, Int. J. Theor. Phys. 35 (1996) 1027-1032.

[3] F. O. Zengin, Math. Rep. 4 (2012) 363-370.

[4] S. Güler and S. A. Demirbağ, Int. J. Theor. Phys. 55 (2016) 548-562.

[5] K. Arslan, R. Deszcz, R. EZENTAŞ, M. Hotlos and C. Murathan, Turkish J. Math. 38 (2014) 353-373.

[6] J. K. Beem and P. E. Ehrlich, Global lorentzian geometry, volume 67 of pure and applied mathematics, Marcel Dekker, New York, 1981.

[7] C. J. S. Clarke, Singularities: global and local aspects, Topological Properties and Global Structure of SpaceTime, Springer, 1986, pp. 61-71.

[8] R. Geroch, Space-time structure from a global viewpoint, General Relativity and Cosmology, vol. 1, 1971, pp. 71103.

[9] S. W. Hawking and G. F. R. Ellis, The large scale structure of space-time, vol. 1, Cambridge university press, 1973.

[10] P. S. Joshi, Int. Ser. Monogr. Phys., Vol. 87, 87 (1993) .

[11] S. Tachibana, Proc. Japan Acad. Ser. Math. Sci. 50 (1974) 301-302.

[12] L. Verstraelen, Geometry and Topology of Submanifolds 6 (1994) 994.

[13] Z. I. Szabó et al., J. Diff. Geom. 17 (1982) 531-582.

[14] Z. I. Szabó, Acta Sci. Math. (Szeged) 47 (1984) 321-348.

[15] Z. I. Szabó, Geometriae Dedicata 19 (1985) 65-108.

[16] O. Kowalski, Czechoslovak Math. J. 46 (1996) 427-474.

[17] E. Boeckx, O. Kowalski and L. Vanhecke, Riemannian manifolds of conullity two, singapore world sci, 1996.

[18] F. Defever, R. Deszcz, L. Verstraelen and L. Vrancken, J. Math. Phys. 35 (1994) 5908-5921.

[19] S. Haesen and L. Verstraelen, J. Math. Phys. 45 (2004) 2343-2346.

[20] R. Deszcz, S. Haesen and L. Verstraelen, Soochow J. Math 30 (2004) 339-349.

[21] V. A. Mirzoyan, Itogi Nauki i Tekhniki. Ser. Probl. Geom.” 23 (1991) 29-66 (in Russian).

[22] B. Y. Chen and K. Yano, Tensor NS 26 (1972) 318-322.

[23] U. C. De and L. Velimirović, Int. J. Theor. Phys. 54 (2015) 1779-1783.

[24] J. Deprez, R. Deszcz and L. Verstraelen, Chinese J. Math. 17 (1989) 51-65.

[25] B. O'neill, Semi-riemannian geometry with applications to relativity, 103, vol. 103, Academic press, 1983.

[26] H. Stephani, D. Kramer, M. MacCallum, C. Hoenselaers and E. Herlt, Exact solutions of Einstein's field equations, 2nd ed. by Hans Stephani, Dietrich Kramer, Malcolm MacCallum, Cornelius Hoenselaers, and Eduard Herlt. Cambridge monographs on mathematical physics. Cambridge, UK: Cambridge University Press, 2003 (2003).

[27] N. Mazumder, R. Biswas and S. Chakraborty, Astrophys. Space Sci 334 (2011) 183-186.

[28] L. Amendola and S. Tsujikawa, Dark energy: theory and observations, Cambridge University Press, 2010.

[29] A. Yildiz, U. C. De and A. Cetinkaya, Proc. Natl. Acad. Sci., India, Sect. A: Phys. Sci. 83 (2013) 239-245.

[30] O. Bertolami, A. A. Sen, S. Sen and P. Silva, Mon. Not. R. Astron. Soc. 353 (2004) 329-337. 